\title{
Ferroelectric Transition in Vinylidene Fluoride and Trifluoroethylene Copolymers Studied by NMR Method II. 52 and 65 mol\% Vinylidene Fluoride Copolymers
}

\author{
Fumiaki IsHII and Akira OdAJIMA* \\ Department of Applied Physics, Faculty of Engineering, \\ Hokkaido University, Sapporo 060, Japan \\ * Department of Electrical Engineering, Hokkaido \\ Institute of Technology, Sapporo 006, Japan
}

(Received May 21, 1990)

\begin{abstract}
NMR derivative line shapes $f^{\prime}(\Delta H)$ in vinylidene fluoride (VDF)/trifluoroethylene (TrFE) copolymers with VDF contents of 52 and $65 \mathrm{~mol} \%$ were measured as a function of the alignment angle $\gamma$ between the drawn axis and magnetic field over a temperature range between 20 and $130^{\circ} \mathrm{C}$. The fine structure of $f^{\prime}(\Delta H)$ and the motional narrowings of the line widths in the each copolymer were investigated by comparing them with those for VDF $72 \mathrm{~mol} \%$ copolymer. In the ferroelectric phase, $f^{\prime}(\Delta \mathrm{H})$ 's in VDF 65 and $72 \mathrm{~mol} \%$ copolymers consisted of three components of narrow, intermediate and broad line widths $\left(\Delta H_{\mathrm{s}}, \Delta H_{\mathrm{m}}\right.$, and $\left.\Delta H_{\mathrm{b}}\right), \Delta H_{\mathrm{m}}$ and $\Delta H_{\mathrm{b}}$ which were due to the TrFE and VDF groups, respectively; $f^{\prime}(\Delta H)$ in the VDF $52 \mathrm{~mol} \%$ copolymer had two components of $\Delta H_{\mathrm{s}}$ and $\Delta H_{\mathrm{b}}$. The value of $\Delta H_{\mathrm{b}}$ was almost equal to $\Delta H_{\mathrm{m}}$ in 65 and $72 \mathrm{~mol} \%$ copolymers. When VDF content decreased, the flip-flop motion of TrFE groups in the VDF $72 \mathrm{~mol} \%$ copolymer changed into rotational motion around the chain axis. Near the Curie temperature, $T_{\mathrm{c}}$ two kinds of trans VDF group motion around the chain axis and TrFE group motion coordinate with the VDF rotational motion disappeared in the VDF $52 \mathrm{~mol} \%$ copolymer. At the $T_{\mathrm{c}}, \Delta H_{\mathrm{b}}$ discontinuity for each copolymer was observed, but the thermal hysteresis of $\Delta H_{\mathrm{b}}$ vs. temperature decreased as VDF content decreased, and was not significant in the VDF $52 \mathrm{~mol} \%$ copolymer. These findings suggest that the ferroelectric phase transition is of the first order above VDF $65 \mathrm{~mol} \%$, and of the first order close to the second order for VDF $52 \mathrm{~mol} \%$.
\end{abstract}

KEY WORDS NMR Line Widths / Narrowing Processes / Vinylidene

Fluoride and Trifluoroethylene Groups / Vinylidene Fluoride content /

Motional Modes / Ferroelectric Phase Transition

Vinylidene fluoride (VDF) and trifluoroethylene (TrFE) copolymer is a ferroelectric of order-disorder type, which exhibits structure phase transition, accompanied by conformational changes between the trans chain in the ferroelectric phase and statistical mixture chain of TGT $\bar{G}$ and $T_{3} G_{3} \bar{G}$ conformers in the paraelectric phase. The phase transition changes from second order to first order with increasing VDF contents of 52 to $80 \mathrm{~mol} \%$, and thermal hysteresis of the phase transition appears more clearly above VDF $65 \mathrm{~mol} \%$. Odajima $^{1}$ and Tashiro et al. ${ }^{2}$ have shown that, when the energy difference of the trans to the gauche form, $\Delta U\left(=U_{\mathrm{tt}}-U_{\mathrm{tg}}\right)>0$, is positive, this transition is first order and its thermal hysteresis becomes larger.

Anomalous increases of the dielectric constant for the copolymers with VDF contents of 52,65 , and $72 \mathrm{~mol} \%$ have been observed in the vicinity of the Curie temperature $T_{\mathrm{c}}$ in rising and falling temperature processes (hereafter, referred to as arrow signs $\uparrow$ and $\downarrow$, respectively)..$^{3,4,5}$ If the critical fluctuation of polarization takes place near $T_{\mathrm{c}}$, anomalous increase of the dielectric relaxation strength $\Delta \varepsilon$ 
and critical slowing down of the relaxation time $\tau$ are expected to be maximal for the VDF $52 \mathrm{~mol} \%$ copolymer since the phase transition is second-order. However, anomalies of $\Delta \varepsilon$ and $\tau$ in the copolymers increased progressively with VDF 72,52 , and $65 \mathrm{~mol} \%$. Ohuchi et al. ${ }^{6)}$ suggest that the motion of the electrical chain dipoles, with a relaxation time $\tau_{0}$, may be associated with the thermal conformation fluctuation of certain segments at boundary regions between the trans and statistical mixture segments near $T_{\mathrm{c}}$. The number of segments becomes larger in the following order: $65>52>72 \mathrm{~mol} \%$. However, further reports have not been specially focused on the dependence of VDF content in $\Delta \varepsilon$ and $\tau$.

In a previous paper, ${ }^{7}$ we investigated the ferroelectric phase transition mechanism for the drawn film of the VDF/TrFE copolymer with a VDF content of $72 \mathrm{~mol} \%$, on the basis of the motional characteristics of the chain in the ferroelectric and paraelectric phases, by using NMR method. In the ferroelectric phase, the observed NMR derivative line shape $f^{\prime}(\Delta H)$ consisted of three components of narrow, intermediate and broad line widths $\left(\Delta H_{\mathrm{s}}\right.$, $\Delta H_{\mathrm{m}}$, and $\left.\Delta H_{\mathrm{b}}\right) . \Delta H_{\mathrm{m}}$ and $\Delta H_{\mathrm{b}}$ were attributed to the TrFE and VDF groups in the trans chain in the ferroelectric crystalline region, respectively. $\Delta H_{\mathrm{s}}$ was due to the amorphous region, whose fraction was 0.05 . In the paraelectric phase, $f^{\prime}(\Delta H)$ consisted of two components of narrow and broad line width $\left(\Delta H_{\mathrm{s}}\right.$ and $\left.\Delta H_{\mathrm{b}^{\prime}}\right)$, of which $\Delta H_{\mathrm{b}}$ was attributed to the statistical mixture chain of TGT $\bar{G}$ and $\mathrm{T}_{3} \mathrm{GT}_{3} \overline{\mathrm{G}}$ conformers in the crystalline region ${ }^{2} . \Delta H_{\mathrm{m}}$, $\Delta H_{\mathrm{b}}$, and $\Delta H_{\mathrm{b}^{\prime}}$ depended on the alignment angle $\gamma$ between the drawn direction and magnetic field.

The motional narrowing of the NMR second moment $\left\langle\Delta H^{2}\right\rangle$ was observed in the four temperature regions of the $\uparrow$-process. The motion narrowing processes in the respective regions were referred to as $\alpha_{\mathrm{m}}, \alpha_{\mathrm{b}}\left(\beta_{\mathrm{m}}\right), \alpha_{\mathrm{t}}$, and $\alpha_{b^{\prime}}$. The broadening of $\left\langle\Delta H^{2}\right\rangle$ took place in the three temperature regions of the $\downarrow$-process; the broadening processes in respective region were referred to as $\alpha_{b^{\prime}}, \alpha_{t}$, and $\alpha_{\mathrm{m}}$. The variation of the second moment, $\delta\left\langle\Delta H^{2}\right\rangle$, taking place in each temperature region, showed strong $\gamma$-dependence. In the $\alpha_{t} \uparrow$ and $\alpha_{t} \downarrow$ regions, narrowing temperatures of $\left\langle\Delta H^{2}\right\rangle$ equaled the Curie temperatures of $T_{\mathrm{c}} \uparrow$ and $T_{\mathrm{c}} \downarrow$, respectively, which were obtained from DSC and dielectric measurements. The $\delta\left\langle\Delta H^{2}\right\rangle$ against $\gamma$ in the respective $\alpha_{t}$ regions displayed a concave curve. This may be attributed to a motion mode of the VDF segments accompanying the conformational change between the all-trans form and the gauche form. In the ferroelectric phase, $\delta\left\langle\Delta H^{2}\right\rangle$ against angle $\gamma$ in the $\alpha_{\mathrm{m}}$ region displayed a convex curve, which was explained theoretically as a flip-flop motion of a trans TrFE group between two sites $180^{\circ}$ apart, accompanied by rotational oscillation $40^{\circ}$ in amplitude. In the $\alpha_{\mathrm{b}}\left(\beta_{\mathrm{m}}\right)$ region, $\delta\left\langle\Delta H^{2}\right\rangle$ $v s . \gamma$ curve increased with increasing angle $\gamma$ for the broad-line component, but the curve for the whole line shape was concave. The $\gamma$-dependence of the broad component curve was due to the motional mode in which VDF groups in the trans chain oscillated about the chain axis with an amplitude of $10^{\circ}$. The concaveness of $\delta\left\langle\Delta H^{2}\right\rangle$ vs. $\gamma$ curve for the whole shape was associated with motion broadening of $\Delta H_{\mathrm{m}}$ (referred to as $\beta_{\mathrm{m}}$ ) in the $\alpha_{\mathrm{b}}\left(\beta_{\mathrm{m}}\right)$ region, attributed to motion of the TrFE group, coordinated with rotational motion of the VDF group.

At a higher temperature in the paraelectric phase, the $\gamma$-dependence of NMR line width $\Delta H_{\mathrm{b}^{\prime}}$ was interpreted as one-dimensional diffusion motion of conformational defects along the chain axis. Near $T_{\mathrm{c}}$ in the $\alpha_{\mathrm{b}^{\prime}}$ region, slight increase of $\Delta H_{\mathrm{b}^{\prime}}$ was theoretically analyzed in the random phase approximation of the Ising model. ${ }^{8}$ It was shown that the increase of $\Delta H_{\mathrm{b}^{\prime}}$ is due to cooperative fluctuation of polarization between trans segments having the electric dipole moment and diffuse phase transition of a first order near $T_{\mathrm{c}}{ }^{8}$ 
In this paper, NMR derivative absorption curves $f^{\prime}(\Delta H)$ for the drawn films of the VDF 52 and $65 \mathrm{~mol} \%$ copolymers were measured as functions of $T$ and $\gamma$, and fine structures and motional modes of the chain in the ferroelectric and paraelectric phases are studied by comparing them by those for the VDF $72 \mathrm{~mol} \%$ copolymer. Also discussed is how their dependence on VDF content is associated with the behavior of the ferroelectric phase transition when VDF content decreases from 72 to $52 \mathrm{~mol} \%$.

\section{EXPERIMENTAL}

Samples used were random copolymers of vinylidene fluoride (VDF) and trifluoroethylene (TrFE) with VDF contents of 52 , and $65 \mathrm{~mol} \%$ supplied by Daikin Kogyo Co., Ltd. Hereafter, the copolymer with $x \mathrm{~mol} \%$ VDF content is referred to as either $\mathrm{P}\left(\mathrm{VDF}_{x}\right)$ $\operatorname{TrFE}_{110-x}$ ) or the VDF $x$ mol\% copolymer. Copolymer films were prepared by slowly casting from 2-butanone solutions under atmospheric pressure at room temperature, and were then drawn $\times 6$ uniaxially. The drawn films of $\mathrm{P}\left(\mathrm{VDF}_{52} / \mathrm{TrFE}_{48}\right)$ and $\mathrm{P}\left(\mathrm{VDF}_{65} /\right.$ $\mathrm{TrFE}_{35}$ ) were annealed at $130^{\circ} \mathrm{C}$ for $20 \mathrm{hr}$ and $9 \mathrm{hr}$ while pressed between glass plates. The final draw ratios for $\mathrm{P}\left(\mathrm{VDF}_{65} / \mathrm{TrFE}_{35}\right)$ and $\mathrm{P}\left(\mathrm{VDF}_{52} / \mathrm{TrFE}_{48}\right)$ were 4 and 4.5 , respectively. Procedures were carried out under the same conditions as those used in preparing samples for $T_{1}$ measurements. ${ }^{9,10}$

The NMR line shapes in both the copolymers were measured for $\gamma=0,45$, and $90^{\circ}$ by a wide line NMR spectrometer, operating at $40 \mathrm{MHz}$ over a temperature range between 20 and $130^{\circ} \mathrm{C}$.

\section{RESULTS}

\section{NMR Derivative Line Shapes}

Figure $1 \mathrm{a}$ and $\mathrm{b}$ show the right halves of the NMR derivative curves $f^{\prime}(\Delta H)$ for $\mathrm{P}\left(\mathrm{VDF}_{52} /\right.$ $\left.\mathrm{TrFE}_{48}\right), \mathrm{P}\left(\mathrm{VDF}_{65} / \mathrm{TrFE}_{35}\right)$, and $\mathrm{P}\left(\mathrm{VDF}_{72} /\right.$
$\left.\mathrm{TrFE}_{28}\right)$ at 30 and $125^{\circ} \mathrm{C}$ in the $\uparrow$-process for

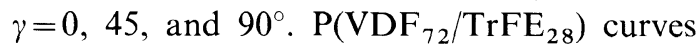
have been reported in the previous paper. ${ }^{7}$ For $\gamma=0$ and $90^{\circ}$ and $30^{\circ} \mathrm{C}, f^{\prime}(\Delta H)$ for $\mathrm{P}\left(\mathrm{VDF}_{52} /\right.$ $\mathrm{TrFE}_{48}$ ) consists of two peaks, which correspond to broad and narrow widths $\left(\Delta H_{\mathrm{b}}\right.$ and $\left.\Delta H_{\mathrm{s}}\right)$. On the other hand, the $f^{\prime}(\Delta H)$ 's for $\mathrm{P}\left(\mathrm{VDF}_{65} / \mathrm{TrFE}_{35}\right)$ and $\mathrm{P}\left(\mathrm{VDF}_{72} / \mathrm{TrFE}_{28}\right)$ consist of three peaks, which correspond to broad, intermediate and narrow widths $\left(\Delta H_{\mathrm{b}}\right.$, $\Delta H_{\mathrm{m}}$, and $\left.\Delta H_{\mathrm{s}}\right)$. However, $f^{\prime}(\Delta H)$ for $\gamma=45^{\circ}$ in $\mathrm{P}\left(\mathrm{VDF}_{65} / \mathrm{TrFE}_{35}\right)$ shows two peaks of narrow and broad widths. The broad width component seems to be made up of $\Delta H_{\mathrm{b}}$ and $\Delta H_{\mathrm{m}}$, although they are not separated. In Figure $1 b$, all $f^{\prime}(\Delta H)$ 's show two peaks of narrow and broad widths $\left(\Delta H_{\mathrm{s}^{\prime}}\right.$ and $\left.\Delta H_{\mathrm{b}^{\prime}}\right)$, at $125^{\circ} \mathrm{C}$ for $\gamma=0,45$, and $90^{\circ} . \Delta H_{\mathrm{b}}$ and $\Delta H_{\mathrm{b}^{\prime}}$, for all copolymers, and $\Delta H_{\mathrm{m}}$ for VDF 65 and $72 \mathrm{~mol} \%$ copolymers, depend on angle $\gamma$, while those for $\Delta H_{\mathrm{s}}$ and $\Delta H_{\mathrm{s}^{\prime}}$ do not. The narrow component fraction in $f^{\prime}(\Delta H)$, observed over the temperature range between 30 and $130^{\circ} \mathrm{C}$, was approximately $4 \%$ for VDF $52 \mathrm{~mol} \%$ copolymer and $6 \%$ for the VDF $65 \mathrm{~mol} \%$ copolymer. The fractions were evaluated by the Willson and Pake' mothod. ${ }^{11}$

\section{Line Width and Its Temperature Dependence}

Figure $2 \mathrm{a}$ and $\mathrm{b}$ show the line widths in $\mathrm{P}\left(\mathrm{VDF}_{65} / \mathrm{TrFE}_{35}\right)$, plotted against temperature, for $\gamma=0$ and 45 , and $90^{\circ}$. In the temperature region between 50 and $85^{\circ} \mathrm{C}$ for the $\uparrow$ - and $\downarrow$-processes, the $\Delta H_{\mathrm{b}}$ component appeared as the shoulder of the intermediate component in the respective line shapes for $\gamma=0$ and $90^{\circ}$. Therefore, line width value were plotted as $\bigcirc$ and $\circlearrowleft$ for the $\uparrow$ - and $\downarrow$-processes, respectively; These corresponded to maximum point of the shoulder.

In the $\uparrow$-process, $\Delta H_{\mathrm{b}} \mathrm{s}$ for $\gamma=0$ and $90^{\circ}$ narrow abruptly in temperature regions between 80 and $90^{\circ} \mathrm{C}$ and between 70 and $80^{\circ} \mathrm{C}$, respectively, and then narrow slightly up to $125^{\circ} \mathrm{C}$. The respective narrowing processes of $\Delta H_{\mathrm{b}}$ for each angle are referred to as $\alpha_{\mathrm{t}}(\uparrow)$ and $\alpha_{b^{\prime}}(\uparrow)$, respectively, in the order of increasing 

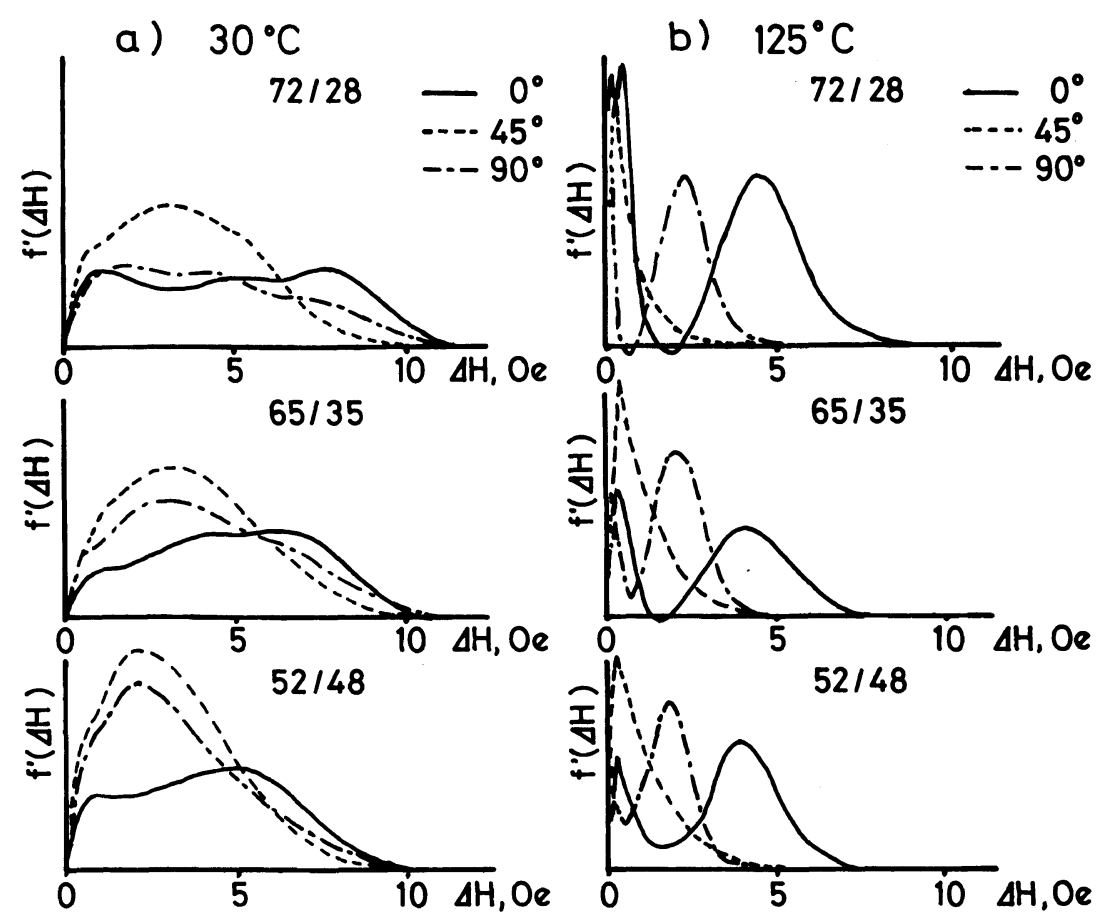

Figure 1. Derivative NMR absorption line shapes of VDF/TrFE copolymers with VDF content of 52 , 65 , and $72 \mathrm{~mol} \%$ for 0,45 , and $90^{\circ}$ of the alignment angle between the drawn axis and the magnetic field; (a) $30^{\circ} \mathrm{C}$ and (b) $125^{\circ} \mathrm{C}$.

temperature. On the other hand, $\Delta H_{\mathrm{b}} \mathrm{s}$ for $\gamma=45^{\circ}$ decrease between 40 and $70^{\circ} \mathrm{C}$ and between 75 and $85^{\circ} \mathrm{C}$.

$\Delta H_{\mathrm{m}} \mathrm{s}$ for $\gamma=0$ and $90^{\circ}$ gradually decrease by about 3 Gs between 40 and $70^{\circ} \mathrm{C}$ and by 2 Gs between 35 and $65^{\circ} \mathrm{C}$, respectively, and then increase gradually by $1-2 \mathrm{Gs}$ up to $105^{\circ} \mathrm{C}$. $\Delta H_{\mathrm{m}}$ seems to approach $\Delta H_{\mathrm{b}^{\prime}}$ above $100^{\circ} \mathrm{C}$, except for $\gamma=45^{\circ}$. The narrowing and broadening processes of $\Delta H_{\mathrm{m}}$ for each angle $\gamma$ are referred to as $\alpha_{\mathrm{m}}(\uparrow)$ and $\beta_{\mathrm{m}}(\uparrow)$, respectively, in the order of increasing temperature. The temperature regions of $\alpha_{x}(x=\mathrm{m}, \mathrm{b}$, t, etc.) and $\beta_{\mathrm{m}}$ are referred to as $\alpha_{x}$ and $\beta_{\mathrm{m}}$ regions, respectively. Since $\alpha_{\mathrm{b}}(\uparrow)$ and $\beta_{\mathrm{m}}(\uparrow)$ processes take place in the same temperature region, their temperature region is referred to as $\beta_{\mathrm{m}}\left(\alpha_{\mathrm{b}}\right)$.

Broadening of $0.5-1.2$ Gs was observed for $\Delta H_{\mathrm{b}^{\prime}}$ between 125 and $80^{\circ} \mathrm{C}$ in the $\downarrow$-process, which is referred to as $\alpha_{b^{\prime}}(\downarrow)$. The line width (O) extremely broadens between 78 and $74^{\circ} \mathrm{C}$ and then returns to the value of $\Delta H_{\mathrm{b}}$ in the $\uparrow$-process. The intermediate width simultaneously appears at $69^{\circ} \mathrm{C}$, and decreases between 69 and $50^{\circ} \mathrm{C}$; it then returns to the value of $\Delta H_{\mathrm{m}}$ in the $\alpha_{\mathrm{m}}$ region of the $\uparrow$-process. Extreme broadening of $\Delta H_{\mathrm{b}^{\prime}}$ and narrowing of $\Delta H_{\mathrm{m}}$ in the $\downarrow$-process are referred to as $\alpha_{\mathrm{t}}(\downarrow)$ and $\beta_{\mathrm{m}}(\downarrow)$, respctively. The line width of $\Delta H_{\mathrm{b}^{\prime}}$ for $\gamma=45^{\circ}$ broadens gradually in the $\alpha_{b^{\prime}}(\downarrow)$ region and increases abruptly in the $\alpha_{t}(\downarrow)$ region, and then returns to the value of the $\uparrow$-process.

Figure 3 shows the line width in $\mathrm{P}\left(\mathrm{VDF}_{52} /\right.$ $\mathrm{TrFE}_{48}$ ), plotted against temperature, for $\gamma=0$, 45 , and $90^{\circ}$. In the $\uparrow$-process, line widths $\Delta H_{\mathrm{b}} \mathrm{s}$ for $\gamma=0,45$, and $90^{\circ}$ narrow gradually between 40 and $60^{\circ} \mathrm{C}$, decrease abruptly between 60 and $70^{\circ} \mathrm{C}$, and then decrease slightly between 70 and $125^{\circ} \mathrm{C}$. The narrowing processes of $\Delta H_{\mathrm{b}}$ in the $\uparrow$-process are referred to as $\alpha_{\mathrm{m}}, \alpha_{\mathrm{t}}$, and $\alpha_{b^{\prime}}$, respectively, in the order of increasing temperature. The temperature region of the $\alpha_{x}$ 

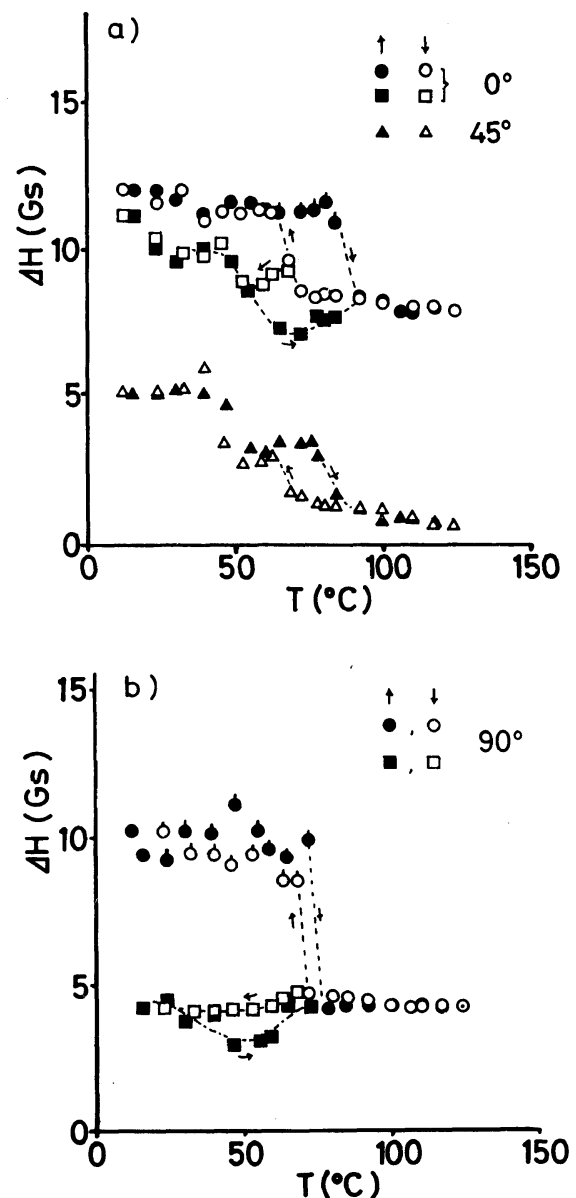

Figure 2. Line widths of $\mathrm{P}\left(\mathrm{VDE}_{65} / \mathrm{TrFE}_{35}\right)$ as a function of temperature: (a) $\gamma=0$ and $45^{\circ}$; (b) $\gamma=90^{\circ}$. The filled and unfilled marks of circle and triangle indicate the broad widths in the $\uparrow$ - and $\downarrow$-processes, respectively and those of square indicate the intermediate widths in the $\uparrow$ - and $\downarrow$-processes, respectively.

$\left(x=\mathrm{m}, \mathrm{t}, \mathrm{b}^{\prime}\right)$ is referred to as $\alpha_{x}$ region. It should be noted that $\Delta H_{\mathrm{b}} \mathrm{s}$ for $\gamma=0^{\circ}$ discontinuously decrease in the $\alpha_{t}$ region, although decrement of $\Delta H_{\mathrm{b}}$ is smaller those of the other angles. In the $\downarrow$-process, $\Delta H_{\mathrm{b}} \mathrm{s}$ increase in the same three temperature regions as $\alpha_{b^{\prime}}, \alpha_{t}$, and $\alpha_{m}$ in the $\uparrow$-process; These $\Delta H_{\mathrm{b}^{\prime}}$ s show reversible changes under $\uparrow$ - and $\downarrow$-conditions.

The $\alpha_{\mathrm{m}} \uparrow, \beta_{\mathrm{m}}\left(\alpha_{\mathrm{b}}\right), \alpha_{\mathrm{t}} \uparrow, \alpha_{\mathrm{t}} \downarrow, \alpha_{\mathrm{b}^{\prime}} \uparrow, \alpha_{\mathrm{b}^{\prime}} \downarrow, \alpha_{\mathrm{t}} \downarrow, \beta_{\mathrm{m}} \downarrow$, and $\alpha_{\mathrm{m}} \downarrow$ regions for $\mathrm{P}\left(\mathrm{VDF}_{65} / \mathrm{TrFE}_{35}\right)$, and the $\alpha_{m}, \alpha_{t}$ and $\alpha_{b^{\prime}}$ regions in the $\uparrow$ - and $\downarrow$-processes for $\mathrm{P}\left(\mathrm{VDF}_{52} / \mathrm{TrFE}_{48}\right)$ are respectively sum-

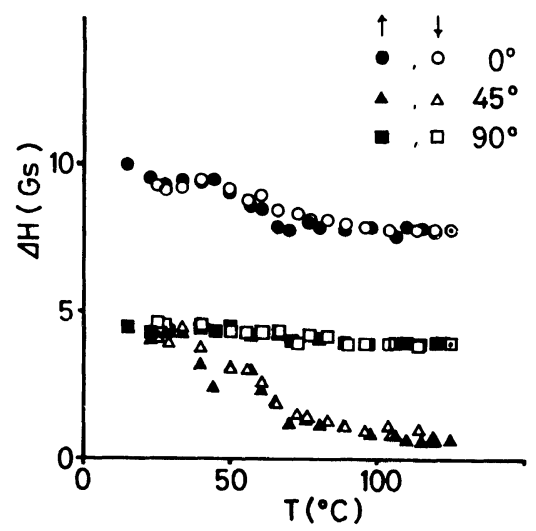

Figure 3. Line widths of $\mathrm{P}\left(\mathrm{VDF}_{52} / \mathrm{TrFE}_{48}\right)$ as a function of temperature in the $\uparrow$ - and $\downarrow$-processes. Line width values for $\gamma=0^{\circ}$ are indicated by filled circle in the $\uparrow$-process and unfilled circle in the $\downarrow$-process $(\bigcirc, \bigcirc)$; those for $\gamma=45^{\circ}$ are indicated by triangles $(\Delta, \triangle)$; those for $\gamma=90^{\circ}$ are indicated by square $(\square, \square)$.

Table I. Classification of motion states and transition temperatures for $\mathrm{P}\left(\mathrm{VDF}_{65} / \mathrm{TrFE}_{35}\right)$

\begin{tabular}{|c|c|c|c|}
\hline \multicolumn{2}{|c|}{$\uparrow$-process } & \multicolumn{2}{|c|}{$\downarrow$-process } \\
\hline \multirow[t]{2}{*}{$\begin{array}{l}\text { Motion } \\
\text { state }\end{array}$} & $\begin{array}{l}\text { Temperature } \\
\text { region }\end{array}$ & $\begin{array}{c}\text { Motion } \\
\text { state }\end{array}$ & $\begin{array}{c}\text { Temperature } \\
\text { region }\end{array}$ \\
\hline & $\mathrm{C}$ & & "C \\
\hline$x_{m} \uparrow$ & $40-70$ & $\alpha_{m} \downarrow$ & $50-40$ \\
\hline$\beta_{\mathrm{m}}\left(\alpha_{\mathrm{b}}\right) \uparrow$ & $70-80$ & $\beta_{\mathrm{m}} \downarrow$ & $69-50$ \\
\hline$\alpha_{1} \uparrow$ & $80-90$ & $\alpha_{1} \downarrow$ & $78-74$ \\
\hline$\alpha_{b}, \uparrow$ & $90-110$ & $\alpha_{b} \downarrow$ & $110-78$ \\
\hline
\end{tabular}

Table II. Classification of motion states and transition temperatures for $\mathrm{P}\left(\mathrm{VDF}_{52} / \mathrm{TrFE}_{48}\right)$

$\uparrow$-process $\downarrow$-process

\begin{tabular}{cccc}
$\begin{array}{c}\text { Motion } \\
\text { state }\end{array}$ & $\begin{array}{c}\text { Temperature } \\
\text { region }\end{array}$ & $\begin{array}{c}\text { Motion } \\
\text { state }\end{array}$ & $\begin{array}{c}\text { Temperature } \\
\text { region }\end{array}$ \\
\cline { 2 - 2 } & $\mathrm{C}$ & & $\mathrm{C}$ \\
& & & $60-40$ \\
$\alpha_{\mathrm{m}} \uparrow$ & $40-60$ & $\alpha_{m} \downarrow$ & $70-60$ \\
$\alpha_{1} \uparrow$ & $60-70$ & $\alpha_{1} \downarrow$ & $110-70$ \\
$\alpha_{\mathrm{b}^{\prime}} \uparrow$ & $70-110$ & $\alpha_{\mathrm{b}^{\prime} \downarrow}$ &
\end{tabular}



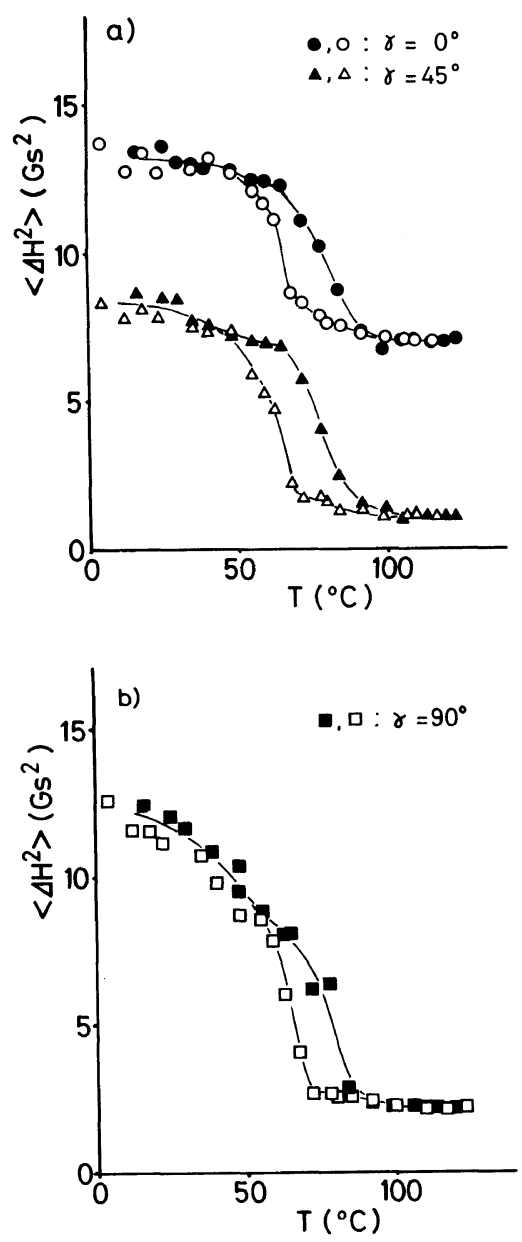

Figure 4. Temperature changes of the NMR second moments, $\left\langle\Delta H^{2}\right\rangle(\gamma)$ 's for $\mathrm{P}\left(\mathrm{VDF}_{65} / \mathrm{TrFE}_{35}\right)$ : (a) values of $\left\langle\Delta H^{2}\right\rangle$ in the $\uparrow$ - and $\downarrow$-processes are indicated, respectively, by the filled and unfilled marks of $\boldsymbol{0}, \bigcirc$ for $\gamma=0^{\circ}$, and those of $\boldsymbol{\Delta}, \triangle$ for $\gamma=45^{\circ}$; (b) filled and unfilled squares of $\square, \square$ are the values of $\left\langle\Delta H^{2}\right\rangle$ for $\gamma=90^{\circ}$ in the $\uparrow$ - and $\downarrow$-processes, respectively.

marized in Tables I and II.

\section{NMR Second Moment and Mobile Fraction}

Figure $4 \mathrm{a}$ and $\mathrm{b}$ show temperature changes of the NMR second moments, $\left\langle\Delta H^{2}\right\rangle(\gamma) \mathrm{s}$ in $\mathrm{P}\left(\mathrm{VDF}_{65} / \mathrm{TrFE}_{35}\right)$ for $\gamma=0,45$, and $90^{\circ}$. The $\left\langle\Delta H^{2}\right\rangle_{\mathrm{S}}$ for $\gamma=0^{\circ}$ in the $\uparrow$ - and $\downarrow$-processes respectively are indicated by filled and unfilled circles $(\bigcirc, \bigcirc)$; those of for $\gamma=45^{\circ}$ are indicated by triangles $(\boldsymbol{\Delta}, \triangle)$ and those for $\gamma=90^{\circ}$ by
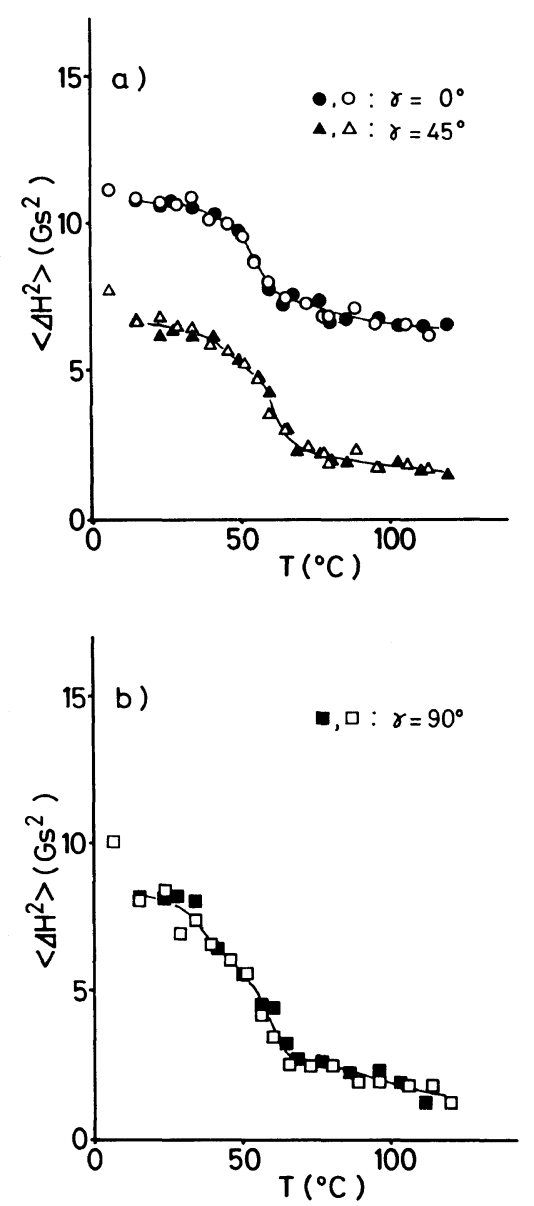

Figure 5. Temperature changes of the NMR second

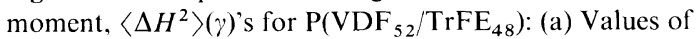
$\left\langle\Delta H^{2}\right\rangle$ in the $\uparrow$ - and $\downarrow$-processes are indicated respectively by the filled and unfilled marks of $\bigcirc, \bigcirc$ for $\gamma=0^{\circ}$, and those of $\boldsymbol{\Delta}, \triangle$ for $\gamma=45^{\circ}$; (b) filled and unfilled squares of $\square, \square$ are the values of $\left\langle\Delta H^{2}\right\rangle$ for $\gamma=90^{\circ}$ in the $\uparrow$ - and $\downarrow$-processes, respectively.

squares $(\boldsymbol{\square}, \square)$. In the $\uparrow$-process, $\left\langle\Delta H^{2}\right.$ ) for $\gamma=0,45$, and $90^{\circ}$ decreases gradually in the $\alpha_{\mathrm{m}}$ region and extremely in the wide temperature range over the $\beta_{\mathrm{m}}\left(\alpha_{\mathrm{b}}\right), \alpha_{\mathrm{t}}$, and $\alpha_{\mathrm{b}^{\prime}}$ regions. The latter narrowing process is referred to as $\alpha_{t}^{\prime}(\uparrow)$. The $\alpha_{t}^{\prime}(\uparrow)$ s for $\gamma=45$ and $90^{\circ}$ take place in a $5-10^{\circ} \mathrm{C}$ lower temperature region than the $\alpha_{t}^{\prime}$ region for $\gamma=0^{\circ}$. When temperature falls, $\left\langle\Delta H^{2}\right\rangle$ for each angle $\gamma$ increases slightly $1-3$ Gs in the $\alpha_{b^{\prime}}$, region. Furthermore, the values of $\left\langle\Delta H^{2}\right\rangle$ increase abruptly in $\alpha_{t}^{\prime}(\downarrow)$ region and 
return to $\left\langle\Delta H^{2}\right\rangle$ in the $\alpha_{\mathrm{m}}$ region of the $\uparrow$-process. Decrements of $\left\langle\Delta H^{2}\right\rangle, \delta\left\langle\Delta H^{2}\right\rangle$ in the $\alpha_{\mathrm{m}}$ and $\alpha_{\mathrm{t}}$, regions depend on the angle $\gamma$. The fraction, $F_{\mathrm{m}}$ of the narrow and intermediate components increased by 12 and $40 \%$ in the $\alpha_{\mathrm{m}}$ and $\beta_{\mathrm{m}}\left(\alpha_{\mathrm{b}}\right)$ regions, respectively.

Figure 5a and $\mathrm{b}$ show the temperature changes of the NMR second moment, $\left\langle\Delta H^{2}\right\rangle(\gamma)$ for $\mathrm{P}\left(\mathrm{VDF}_{52} / \mathrm{TrFE}_{48}\right) .\left\langle\Delta H^{2}\right\rangle_{\mathrm{S}}$ in the $\uparrow$ - and $\downarrow$-processes respectively are indicated by filled and unfilled circles $(0, \bigcirc)$; those of for $\gamma=45^{\circ}$ are indicated by triangles $(\boldsymbol{\Delta}, \triangle)$ and those for $\gamma=90^{\circ}$ by squares $(\square, \square)$. The $\left\langle\Delta H^{2}\right\rangle v s$. $T$ curve for each $\gamma$ decreases in three stages over the wide temperature range of the $\alpha_{\mathrm{m}}, \alpha_{\mathrm{t}}$ and $\alpha_{b}^{\prime}$ regions. Thermal hysteresis does not take place in these $\uparrow$ - and $\downarrow$-processes. $\delta\left\langle\Delta H^{2}\right\rangle$ in each temperature region increases with increasing angle $\gamma$. The $F_{\mathrm{m}}$ of the narrow component decreases 3 to $4 \%$ in the $\alpha_{\mathrm{t}}$ region.

\section{DISCUSSION}

Line width estimation of $f^{\prime}(\Delta H)$ depends on $V D F$ content

In Figure 1, comparing $\Delta H_{\mathrm{m}}$ and $\Delta H_{\mathrm{b}}$ of $f^{\prime}(\Delta H)$ in $\mathrm{P}\left(\mathrm{VDF}_{65} / \mathrm{TrFE}_{35}\right)$ for each $\gamma$ with those of $\mathrm{P}\left(\mathrm{VDF}_{72} / \mathrm{TrFE}_{28}\right), \Delta H_{\mathrm{b}}$ and its component fractions in $f^{\prime}(\Delta H)$ decrease; $\Delta H_{\mathrm{m}}$ however does not change significantly, though its component fraction increases. For $\mathrm{P}\left(\mathrm{VDF}_{52} /\right.$ $\left.\operatorname{TrFE}_{48}\right), \Delta H_{\mathrm{b}}$ is similar to the value of $\Delta H_{\mathrm{m}}$ for the TrFE group in $\left.\mathrm{P}\left(\mathrm{VDF}_{72} / \mathrm{TrFE}_{28}\right)\right)^{7}$ These findings suggest that $\Delta H_{\mathrm{m}}$ for the VDF $65 \mathrm{~mol} \%$ copolymer and $\Delta H_{\mathrm{b}}$ for the VDF $52 \mathrm{~mol} \%$ copolymer may be attributed to the TrFE groups of the trans chain in the ferroelectric phase. At $125^{\circ} \mathrm{C}$, the value of $\Delta H_{\mathrm{b}^{\prime}}$ in $f^{\prime}(\Delta H)$ at each angle $\gamma$ has the same value for all copolymers, which may be attributed to fluctuation of the local magnetic field which is modulated by the one-dimensional diffusion motion of the conformational defects in the paraelectric phase.
Molecular Motions in Ferroelectric and Paraelectric Phases

The thermal hystereses of $\Delta H_{\mathrm{b}}(\gamma)$ and $\left\langle\Delta H^{2}\right\rangle(\gamma)$ in $\mathrm{P}\left(\mathrm{VDF}_{x} / \operatorname{TrFE}_{100-x}\right)$ become smaller in the order of decreasing VDF content $x$, from 72 to $52 \mathrm{~mol} \%$, as shown in Figures $2-5$. The abrupt change of the broad line width between $\Delta H_{\mathrm{b}}$ and $\Delta H_{\mathrm{b}}$, in each copolymer for $\gamma=0^{\circ}$ takes place in the $\alpha_{\mathrm{t}} \uparrow$ and $\alpha_{\mathrm{t}} \downarrow$ regions as shown in Figures 2 and 3, whereas the change in $f^{\prime}(\Delta H)$, as shown in Figure $1 \mathrm{a}$ and $\mathrm{b}$, took place discontinuously. The narrowing temperatures of $\Delta H_{\mathrm{b}}$ which changed discontinuously in the $\alpha_{t}$ region are listed in Table III as $T_{\mathrm{c}}(\uparrow)$ and $T_{\mathrm{c}}(\downarrow)$ for the $\uparrow$ - and $\downarrow$-processes. Narrowing temperatures for $\mathrm{P}\left(\mathrm{VDF}_{72} / \mathrm{TrFE}_{28}\right)$ were reported in a previous paper. ${ }^{7}$ Both $T_{\mathrm{c}}(\uparrow)$ and $T_{\mathrm{c}}(\downarrow)$ shift to lower temperatures when VDF content decreases. They approximate the corresponding transition temperatures obtained from dielectric, ${ }^{4}$ DSC, ${ }^{4}$ and spin lattice relaxation time measurements. ${ }^{9,10}$ The narrowings of $\Delta H_{\mathrm{b}}$ in the $\alpha_{\mathrm{t}}$ region for the VDF 52 and $65 \mathrm{~mol} \%$ copolymers may also be due to ferroelectric phase transition. The discontinuous variation of $\Delta H_{\mathrm{b}}$ in each $\alpha_{\mathrm{t}}$ region and its thermal hysteresis decrease in the order of VDF 75 and $65 \mathrm{~mol} \%$. In the $52 \mathrm{~mol} \%$ copolymer the latter is not significant, but the former occurs slightly at $T_{\mathrm{c}}=63^{\circ} \mathrm{C}$. Such dependences of VDF content in the discontinuous change of $\Delta H_{\mathrm{b}}$ and its thermal hysteresis show that

Table III. Temperatures of $\alpha_{1}$ processes for three VDF/TrFE copolymers with the VDF contents of 52,65 and $72 \mathrm{~mol} \%$ in heating and cooling processes

\begin{tabular}{|c|c|c|c|c|c|c|}
\hline & \multicolumn{2}{|c|}{$52 / 48$} & \multicolumn{2}{|c|}{$65 / 35$} & \multicolumn{2}{|c|}{$72 / 28$} \\
\hline & $T_{\mathrm{c}}(\uparrow)$ & $T_{\mathrm{c}}(\downarrow)$ & $T_{\mathrm{c}}(\uparrow)$ & $T_{\mathrm{c}}(\downarrow)$ & $T_{\mathrm{c}}(\uparrow)$ & $T_{c}(\downarrow)$ \\
\hline & \multicolumn{2}{|c|}{$\mathrm{C}$} & \multicolumn{2}{|c|}{$\mathrm{C}$} & \multicolumn{2}{|c|}{$\mathrm{C}$} \\
\hline$\Delta H$ & 63 & 63 & 89 & 70 & 116 & 75 \\
\hline$T_{1}$ & 65 & 65 & 90 & 75 & 115 & 79 \\
\hline DSC & 60 & 62 & 70 & 64 & 114 & 75 \\
\hline $1 / \Delta r$ & 79 & 73 & 102 & 69 & 134 & 84 \\
\hline
\end{tabular}


this phase transition is of the first order for VDF 65 and $72 \mathrm{~mol} \%$, and that, especially, it is first order close to the second order for VDF $52 \mathrm{~mol} \%$.

$\Delta H_{\mathrm{b}}$ for the VDF $52 \mathrm{~mol} \%$ copolymer and $\Delta H_{\mathrm{m}}$ for the VDF $65 \mathrm{~mol} \%$ copolymers narrow extremely at about $50^{\circ} \mathrm{C}$ in the $\alpha_{\mathrm{m}}$ regions, as does the $\Delta H_{\mathrm{m}}$ of $\mathrm{P}\left(\mathrm{VDF}_{72} / \mathrm{TrFE}_{28}\right){ }^{7}$ This means that the narrowing temperature of $\alpha_{\mathrm{m}}$, due to the motion of the TrFE group, does not depend on VDF content, although the temperature range of $\alpha_{\mathrm{m}}$ decreases with decreasing VDF content as shown in Tables I and II.

In a previous paper, ${ }^{7}$ narrowing of $\Delta H_{\mathrm{b}} \mathrm{s}$ and broadening of $\Delta H_{\mathrm{m}}$ in $\mathrm{P}\left(\mathrm{VDF}_{72} / \mathrm{TrFE}_{28}\right)$ in the $\hat{\imath}$-process took place simultaneously between 80 and $110^{\circ} \mathrm{C}$ in the $\alpha_{\mathrm{b}}\left(\beta_{\mathrm{m}}\right)$ region below $T_{\mathrm{c}}(\uparrow)$. These were attributed respectively to the rotational motion of the VDF groups in the trans chain with amplitude of $10^{\circ}$ around the axis and TrFE group motion coordinated with the rotational motion of the VDF group.

As shown in Figure $2, \Delta H_{\mathrm{m}}$ in $\mathrm{P}\left(\mathrm{VDF}_{65} /\right.$ $\left.\mathrm{TrFE}_{35}\right)$ broadens slightly in the $\beta_{\mathrm{m}}\left(\alpha_{\mathrm{b}}\right)$ region near $T_{\mathrm{c}}$, as it also does in the $\alpha_{\mathrm{b}}\left(\beta_{\mathrm{m}}\right)$ region for $\mathrm{P}\left(\mathrm{VDF}_{72} / \mathrm{TrFE}_{28}\right){ }^{7}$ The narrowing stages of $\Delta H_{\mathrm{b}}$ and $\left\langle\Delta H^{2}\right\rangle$ in $\mathrm{P}\left(\mathrm{VDF}_{65} / \mathrm{TrFE}_{35}\right)$ are not observed clearly in the $\beta_{\mathrm{m}}\left(\alpha_{\mathrm{b}}\right)$ region below $T_{\mathrm{c}}(\uparrow)$, as shown in Figures 2 and 4 , since the ferroelectric phase transition takes place at $T_{\mathrm{c}}(\uparrow)=90^{\circ} \mathrm{C}$. However, for $\gamma=45$ and $90^{\circ}$, extreme decrease in $\Delta H_{\mathrm{b}}$ and $\left\langle\Delta H^{2}\right\rangle$ takes place, respectively, in a 5 to $10^{\circ} \mathrm{C}$ lower temperature region than the $\alpha_{t}$ narrowing region for $\gamma=0^{\circ}$, where $\Delta H_{\mathrm{b}}$ vs. $T$ curve for $\gamma=0^{\circ}$ hardly changes. These findings suggest that in $\mathrm{P}\left(\mathrm{VDF}_{65} / \mathrm{TrFE}_{35}\right)$ two kinds of $\alpha_{\mathrm{b}}$ motion mechanisms may exist: the rotational motion of the VDF groups around the chain axis and increase of $\Delta H_{\mathrm{m}}$ may be attributed to the TrFE group motion coordinated with the rotational motion of VDF groups in the $\beta_{\mathrm{m}}\left(\alpha_{\mathrm{b}}\right)$ region below $T_{c}(\uparrow)$, as they also do in $\mathrm{P}\left(\mathrm{VDF}_{72} / \mathrm{TrFE}_{28}\right)$. On the other hand, although $\Delta H_{\mathrm{b}}$ in the VDF $52 \mathrm{~mol} \%$ copolymer is due to the TrFE groups, broadening of $\Delta H_{\mathrm{b}}$, which corresponds to $\beta_{\mathrm{m}}$ in other copolymers, is never observed near $T_{\mathrm{c}}(\uparrow)$. The disappearance of $\alpha_{\mathrm{b}}$ and $\beta_{\mathrm{m}}$ may be closely associated with the stability of a trans VDF segment ${ }^{1,2}$ near $T_{\mathrm{c}}(\uparrow)$; This suggests that TrFE group motion, coordinated with the rotational motion of the VDF groups, may play an essential role in stabilizing the trans VDF segment. ${ }^{2,7}$

In the paraelectric phase, as shown in Figures $2-4$, when VDF content decreases, the slight increases of $\Delta H_{\mathrm{b}}$ and $\left\langle\Delta H^{2}\right\rangle$ in the $\alpha_{\mathrm{b}^{\prime}}$ region occur at lower temperatures in company with depression of $T_{\mathrm{c}}(\uparrow)$. They increase in increments, becoming larger with decreasing VDF content. In previous papers, ${ }^{6.7}$ the respective increments of $\Delta H_{\mathrm{b}^{\prime}}$ and inverse spin-lattice relaxation time $1 / T_{1}$, in the $\alpha_{b^{\prime}}$ region for each copolymer, were analyzed theoretically on the basis of the Ising model. It was shown that phase transition diffuseness takes place in the critical region close to $T_{\mathrm{c}}(\uparrow)$, that phase transition region becomes larger with decreasing VDF content, and that polarization fluctuation near $T_{\mathrm{c}}$ increases progressively with VDF 72,52 , and $65 \mathrm{~mol} \%$. The shift of $\alpha_{b^{\prime}}$ to low temperature and temperature increase of the diffuse phase transition region may be attributed to the stability of the VDF trans segment. The energy difference of trans and gauche causes changes from positive to

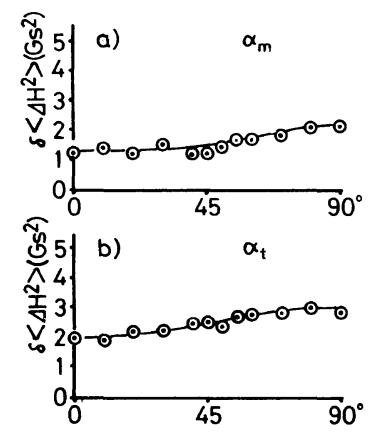

Figure 6. Decrement of the second moment, $\delta\left\langle\Delta H^{2}\right\rangle$ within the following regions of $\mathrm{P}\left(\mathrm{VDF}_{52} / \mathrm{TrFE}_{48}\right)$ as a function of alignment angle $\gamma$ between the drawn direction of the film and the magnetic field: (a) and (b) are the $\delta\left\langle\Delta H^{2}\right\rangle v s . \gamma$ curves in the $\alpha_{\mathrm{m}}$ and $\alpha_{\mathrm{t}}$ regions, respectively. 


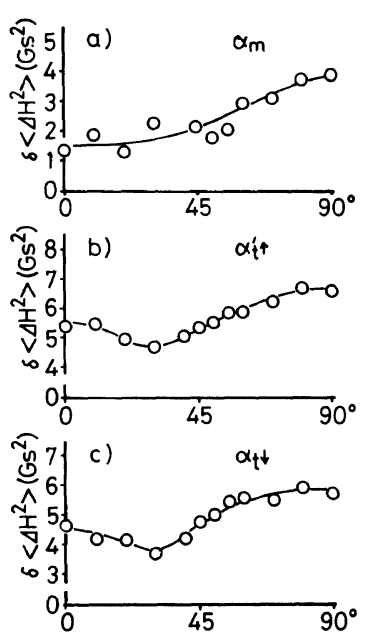

Figure 7. Decrement of the second moment, $\delta\left\langle\Delta H^{2}\right\rangle$ within the following regions of $\mathrm{P}\left(\mathrm{VDF}_{65} / \mathrm{TrFE}_{35}\right)$ as a function of alignment angle $\gamma$ between the drawn direction of the film and the magnetic field; (a) $\alpha_{\mathrm{m}}: \bigcirc, \delta\left\langle\Delta H^{2}\right\rangle$, (b) $\alpha_{1}^{\prime}: \bigcirc, \delta\left\langle\Delta H^{2}\right\rangle$ and (c) $\alpha_{1}: \bigcirc, \delta\left\langle\Delta H^{2}\right\rangle$, respectively.

negative with decreasing VDF content. ${ }^{1,2}$

Figures 6 and 7 show the dependencies of $\delta\left\langle\Delta H^{2}\right\rangle$ on angle $\gamma$ in the $\alpha_{\mathrm{m}}$ and $\alpha_{\mathrm{t}}$ regions for $\mathrm{P}\left(\mathrm{VDF}_{52} / \mathrm{TrFE}_{48}\right)$, and in the $\alpha_{\mathrm{m}}$ and $\alpha_{\mathrm{t}}^{\prime}$ regions for $\mathrm{P}\left(\mathrm{VDF}_{65} / \mathrm{TrFE}_{35}\right)$.

$\delta\left\langle\Delta H^{2}\right\rangle$ vs. $\gamma$ curves in the $\alpha_{t^{\prime}} \uparrow$ and $\alpha_{t}^{\prime} \downarrow$ regions are concave for $\mathrm{P}\left(\mathrm{VDF}_{65} / \mathrm{TrFE}_{35}\right)$ in Figure 7 . They are also concave for $\mathrm{P}\left(\mathrm{VDF}_{72} /\right.$ $\left.\mathrm{TrFE}_{28}\right){ }^{7}$ but for $\mathrm{P}\left(\mathrm{VDF}_{52} / \mathrm{TrFE}_{48}\right)$ they increase with increasing $\gamma$. The concaveness of the $\delta\left\langle\Delta H^{2}\right\rangle$ against $\gamma$ is characteristic of the ferroelectric phase transition of the first order accompanied by conformational change of the VDF segment from trans to gauche. The change of $\delta\left\langle\Delta H^{2}\right\rangle v s . \gamma$ curve in the $\alpha_{\mathrm{t}}$ region, coupled with decrease of VDF content from 65 to $52 \mathrm{~mol} \%$, is associated closely with the appearance of the ferroelectric phase transition which changed from first-order to secondorderlike. This may be attributed to the more stable trans conformation of the VDF segment rather than to the gauche one. ${ }^{1,2}$

$\delta\left\langle\Delta H^{2}\right\rangle$ plotted against the $\gamma$ in the $\alpha_{\mathrm{m}}$ region for the 52 and $65 \mathrm{~mol} \%$ copolymers increased with increasing $\gamma$, in contrast to the convex curve which was maximal at $\gamma=45^{\circ}$ for the
$72 \mathrm{~mol} \%$ copolymer. This change of the $\delta\left\langle\Delta H^{2}\right\rangle v s . \gamma$ curve in the $\alpha_{\mathrm{m}}$ region suggests that the motional mode of the TrFE group changed, under the influence of decreasing VDF content, from flip-flop motion ${ }^{7}$ to rotational motion around the chain axis. This further suggests that the ferroelectric feature of the phase transition depends on VDF content and greatly influences the motion modes of the VDF and TrFE groups in the chain in the ferroelectric phase as well as the disappearances of $\alpha_{\mathrm{b}}$ and $\beta_{\mathrm{m}}$ below $T_{\mathrm{c}}(\uparrow)$ in the VDF $52 \mathrm{~mol} \%$ copolymer.

\section{CONCLUSIONS}

The fine structure, the motion modes of the chain, and ferroelectric phase transition in the copolymers were investigated by comparing NMR line shapes $f^{\prime}(\Delta H)$ and the motional narrowings of the line width $\Delta H$ and the second moment $\left\langle\Delta H^{2}\right\rangle$ in ferroelectric and paraelectric phases for 52 and $65 \mathrm{~mol} \%$ copolymers with those in the VDF $72 \mathrm{~mol} \%$ copolymer. ${ }^{7}$

Below $T_{\mathrm{c}}, f^{\prime}(\Delta H)$ consisted of three components of narrow, intermediate and broad line widths $\left(\Delta H_{\mathrm{s}}, \Delta H_{\mathrm{m}}\right.$, and $\left.\Delta H_{\mathrm{b}}\right)$ for the VDF $65 \mathrm{~mol} \%$ copolymer and two components of, $\Delta H_{\mathrm{s}}$ and $\Delta H_{\mathrm{b}}$, for the VDF $52 \mathrm{~mol} \%$ copolymer. $\Delta H_{\mathrm{s}}$ was due to the amorphous chain. $\Delta H_{\mathrm{b}}$ and $\Delta H_{\mathrm{m}}$ in the ferroelectric phase for the VDF $65 \mathrm{~mol} \%$ copolymer, were attributed to VDF and TrFE groups in the trans chains, respectively, as were those for the VDF $72 \mathrm{~mol} \%$ copolymer. The value of $\Delta H_{\mathrm{b}}$ in $\mathrm{P}\left(\mathrm{VDF}_{52} / \mathrm{TrFE}_{48}\right)$ was equal to the value of $\Delta H_{\mathrm{m}}$ for other copolymers, which was attributed to TrFE groups in the trans chain. Above $T_{\mathrm{c}}, f^{\prime}(\Delta H)$ 's for all copolymers consisted of two components of narrow and broad line widths $\left(\Delta H_{\mathrm{s}}\right.$ and $\left.\Delta H_{\mathrm{b}^{\prime}}\right)$, respectively. $f^{\prime}(\Delta H)$ in the paraelectric phase did not show the fine structure of the VDF and TrFE groups; $\Delta H_{\mathrm{b}^{\prime}}$ was due to the whole statistical chain of TGTG and $\mathrm{T}_{3} \mathrm{GT}_{3} \mathrm{G}$.

The ferroelectric feature of the phase 
transition depended on VDF content and greatly influenced the motion modes of VDF and TrFE groups of the chain in the ferroelectric phase as follows:

(1) The $\alpha_{\mathrm{m}}$ process was attributed to the motion of TrFE groups in the ferroelectric phase; the motion mode of TrFE groups changed from flip-flop motion to a rotational oscillation, when the VDF content decreased from 72 , through 65 and $52 \mathrm{~mol} \%$.

(2) The $\beta_{\mathrm{m}}\left(\alpha_{\mathrm{b}}\right)$ process was attributed to the rotational motion of VDF groups around the trans chain axis $\left(\alpha_{b}\right)$ and motion of TrFE groups, coordinating with the VDF rotational motion $\left(\beta_{\mathrm{m}}\right)$ in the $\beta_{\mathrm{m}}\left(\alpha_{\mathrm{b}}\right)$ region near $T_{\mathrm{c}}$ in conditions of rising temperature. These motions occurred in the VDF 72 and $65 \mathrm{~mol} \%$ copolymers, but did not in the $52 \mathrm{~mol} \%$ copolymer.

(3) The $\alpha_{t}$ process was due to the ferroelectric phase transition accompanied by the conformational change of the VDF segment. The discontinuity of $\Delta H_{\mathrm{b}}$ at $T_{\mathrm{c}}$ and its thermal hysteresis of $\alpha_{t}$ confirmed that the ferroelectric phase transition is first order for VDF 65 and $72 \mathrm{~mol} \%$ copolymers and first order close to second order for the VDF $52 \mathrm{~mol} \%$ copolymer.

The $\alpha_{b^{\prime}}$ process in the paraelectric phase was attributed to the polarization fluctuation of trans segments (order units) near $T_{\mathrm{c}}(\downarrow)$.

Acknowledgments. The authors are grateful to Drs. Jun Koizumi, Junichi Sako, Toshiharu Yagi and Yoshihide Higashihata of Daikin Kyogyo Co., Ltd., for supplying the samples of VDF/TrFE copolymers.

\section{REFERENCES}

1. A. Odajima, Ferroelectrics, 57, 159 (1984).

2. K. Tashiro, T. Takano, M. Kobayashi, Y. Chatani, and H. Tadokoro, Ferroelectrics, 57, 297 (1984).

3. T. Furukawa, M. Ohuchi, A. Chiba, and M. Date, Macromolecules, 17, 1384 (1984).

4. N. Koizumi, N. Haikawa, and H. Habuta, Ferroelectrics 57, 99 (1984).

5. G. T. Davis, T. Furukawa, A. J. Lovinger, and M. G. Broudhurst, Macromolecules, 15, 329 (1982).

6. M. Ohuchi, A. Chiba, A. Data, and T. Furukawa, Jpn. J. Appl. Phys., 22, 1267 (1983).

7. F. Ishii, A. Odajima, and J. Ohigashi, Polym. J., 18, 875 (1983).

8. F. Ishii, A. Tsutsumi, and A. Odajima, Jpn. J. Appl. Phys., 27, 917 (1988).

9. F. Ishii and A. Odajima, Polym. J., 18, 547 (1986).

10. F. Ishii and A. Odajima, Polym. J., 18, 539 (1986).

11. C. W. Willson III and G. E. Pake, J. Polym. Sci., 10, 503 (1953).

12. F. Ishii and A. Odajima, Jpn. J. Appl. Phys., 26, 1641 (1987). 